Key points

- Medical treatment of advanced NSCLC is associated with benefits in terms of palliation, improvement of survival, symptom control, quality of life and cost.

- First-line treatment in the fit patient should be cisplatin-based combination chemotherapy with another active drug, such as gemcitabine, docetaxel, paclitaxel, vinorelbine or pemetrexed.

- Standard dosages of cisplatin are associated with reduced toxicity with similar survival than higher dosages and are thus recommended.

- Cisplatin should be preferred to carboplatin in the fit patient because of a better effect on survival.

- Second-line chemotherapy should be proposed in patients with good performance status, failing after platinum-based first-line chemotherapy. Evidence is in favour of docetaxel; alternatives are pemetrexed (in non-squamous NSCLC) and erlotinib.

- An activating mutation of EGFr, particularly in adenocarcinoma, should be sought and, if present, the patient should be treated by gefitinib, whatever the line of treatment.

- Treatment has to be adapted in case of poor performance status, elderly or organ dysfunction.

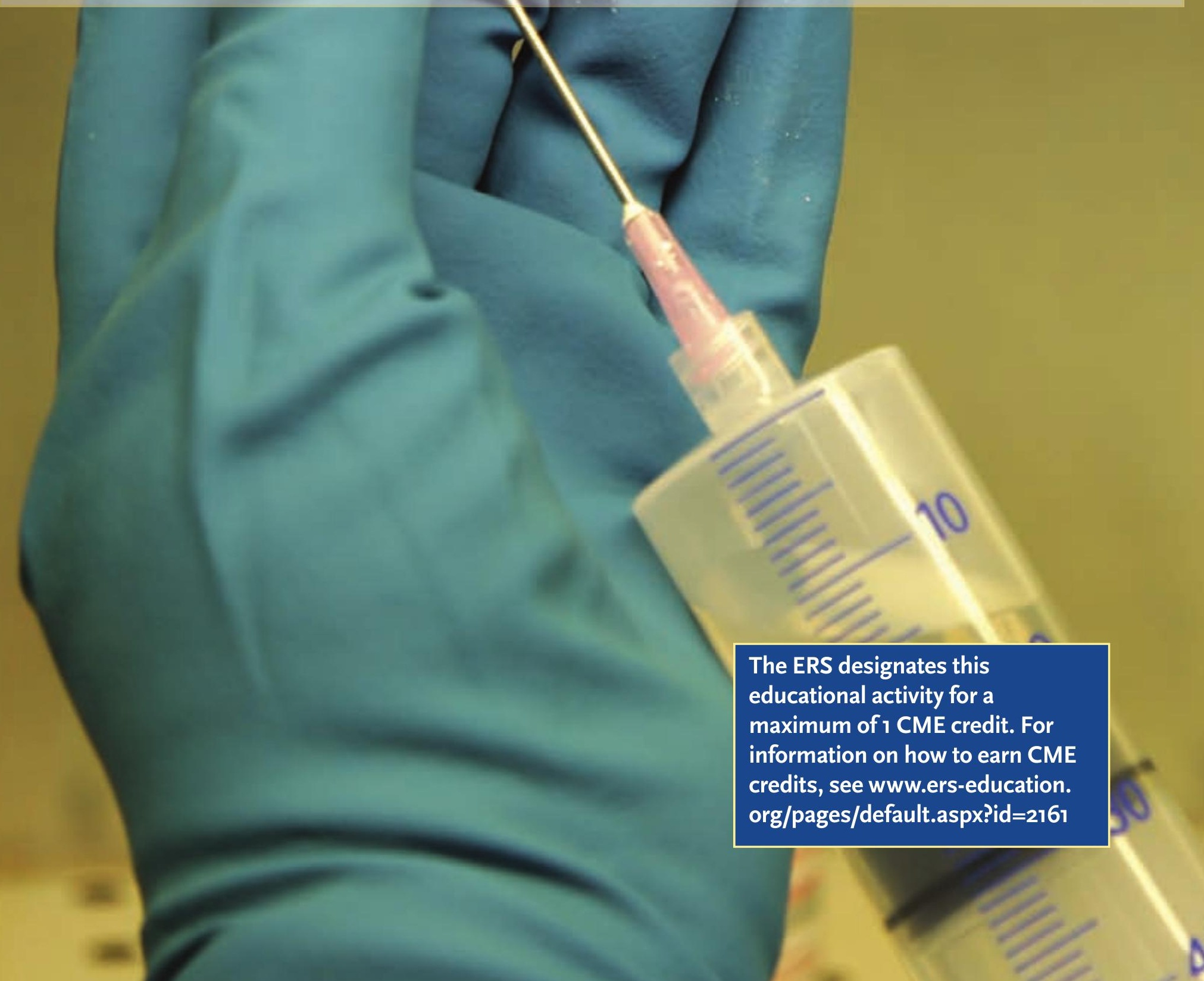




\begin{tabular}{|c|c|c|c|}
\hline$\Omega$ & 0 & $\equiv$ & (르 \\
\hline J-P. Sculier & $\begin{array}{l}\text { Intensive Care \& Thoracic } \\
\text { Oncology, Institut Jules } \\
\text { Bordet, Université Libre de } \\
\text { Bruxelles, Brussels, Belgium }\end{array}$ & $\begin{array}{l}\text { Intensive Care \& Thoracic } \\
\text { Oncology, Institut Jules } \\
\text { Bordet, Université Libre de } \\
\text { Bruxelles (ULB), 1, rue } \\
\text { Héger-Bordet, B-10oo } \\
\text { Bruxelles, Belgium }\end{array}$ & sculier@bordet.be \\
\hline
\end{tabular}

\section{Anticancer treatment for advanced non-small cell lung cancer}

\section{Educational aims}

- To propose a practical approach for the medical treatment of the patient with advanced non-small cell lung cancer, taking into account the fitness or unfitness of the patient for standard chemotherapy.

$\odot$ To provide evidence supporting the recommended approach.

\section{Summary}

Practical management of the patient with advanced non-small cell lung cancer is reviewed. First-line and salvage treatments are discussed as well as special situations, such as elderly, poor performance status and organ dysfunction. Special attention has been given to recently developed drugs that are registered within the European Union.

Advanced non-small cell lung cancer (NSCLC) is defined as a disease that cannot be treated by a curative approach. It corresponds to stages where surgery and/or chest irradiation cannot be performed. Practically, it consists mainly in stage-IV or in some advanced stage-III of the 2009 International Staging System.

In order to optimally manage a patient with advanced NSCLC, the following information is required for the treatment plan: histological type, TNM (tumour, node, metastasis) stage, sites of metastases, performance status, age, existence of comorbidities, and organ (lung, heart, bone marrow, liver) function assessment.
This treatment plan should be proposed after a multidisciplinary discussion including representatives of the following disciplines: pathology, chest medicine, radiation oncology, medical oncology, thoracic surgery, radiology, nuclear medicine and palliative care.

The management of patients with advanced NSCLC has become more sophisticated. Progress in imaging procedures including brain nuclear magnetic resonance (NMR) and positron emission tomography-computed tomography (PET-CT) scan has allowed earlier diagnosis of advanced disease. More anticancer drugs are now available, allowing multiple
Statement of interest None declared. 
lines of treatment. Local treatment of some metastatic sites, such as brain by stereotactic irradiation, is associated with better disease control. Supportive care, including intensive care, improves the treatment of complications. Although it is difficult to demonstrate using clinical trials, all these ameliorations have led to better survival and quality of life.

In routine practice, in many multidisciplinary rounds, treatment is based on a local programme established using published guidelines which are mainly oriented by the results of meta-analyses and randomised trials (see www. pneumocancero.com), using the principles of evidence-based medicine. It is very important for the physician to understand the objectives of those studies which are the background of most clinical practice recommendations. Indeed, a trial can be performed for three main reasons: scientific, clinical and commercial. The scientific objective deals with a better understanding of the treatment effect but this information is usually not directly helpful for the patient management. The clinical objective determines the effect of the treatment in terms of benefits for the patients (survival, quality of life, symptom control). The commercial purpose is a main objective in the studies sponsored by the industry but it is usually not reported and has to be figured out by the physician, requiring some expertise in the field. Today, for the studies conducted in the European Union, Eudrapharm provides the information in the EU Clinical Trials Register, using the terminology of commercial or non-commercial according to the type of sponsorship. The EU Clinical Trials Register is free to access on the internet.
Keeping this introduction in mind and without fully reviewing the literature, which has been the topic of a recent report [1], we will now address the questions that have to be solved in the practical management of patients with advanced NSCLC.

\section{What are the benefits of the medical treatment?}

This is a frequently asked question by the patient. Randomised trials performed in the 1980 s and 1990 s have shown benefits with chemotherapy (mainly cisplatin-based) in terms of palliation, improvement of survival, symptom control, quality of life and cost. Seven metaanalyses published in the 1990s, including one performed with individual patients' data (table 1) which has been recently updated [2], have confirmed a modest but significant effect with chemotherapy in terms of survival.

There will be no more trials in patients fit for chemotherapy to demonstrate its benefits in advanced NSCLC. However, from a clinical point of view, the control arm in new studies should always correspond to a regimen shown, by formal comparison, equivalent or superior to those that have established the survival benefit.

\section{Which drugs are available in Europe?}

The active drugs registered and marketed in the European Union for the treatment of NSCLC (table 2) can be divided in three main groups: 1) the agents with generic forms; 2) the agents

Table 1 Chemotherapy in addition to supportive care in advanced NSCLC: main results of the meta-analysis performed on individual patient data from 16 randomised controlled trials [2]

\begin{tabular}{|c|c|c|c|}
\hline & \multicolumn{2}{|c|}{ Arm } & \\
\hline & Chemotherapy & Supportive care & \\
\hline Patients $n$ & 1399 & 1315 & \\
\hline Deaths $n$ & 1293 & 1240 & \\
\hline Hazard ratio & & & $\begin{array}{l}95 \% \mathrm{Cl}: 0.71-0.83 \\
\mathrm{P}<0.0001\end{array}$ \\
\hline 1-yr survival & $29 \%$ & $20 \%$ & \\
\hline Median survival & 6 months & 4.5 months & \\
\hline
\end{tabular}


Table 2 Active drugs for the treatment of NSCLC

\begin{tabular}{lll}
\hline With generic forms & $\begin{array}{l}\text { With generic forms and } \\
\text { restricted indications }\end{array}$ & Protected by patents \\
\hline Cisplatin & Docetaxel & Pemetrexed \\
\hline Ifosfamide & Bevacizumab \\
\hline Mitomycin C & Erlotinib \\
\hline Vindesine & Gefitinib \\
\hline Vinblastine & \\
\hline Gemcitabine & \\
\hline Paclitaxel & \\
\hline Vinorelbine & \\
\hline Carboplatine & \\
\hline
\end{tabular}

Etoposide

with generic forms but with restricted indications; and 3) the agents protected by patents. In the second group, there is only docetaxel which has been registered for first-line treatment in association with cisplatin and also for salvage chemotherapy. There are only four commercially available drugs protected by a patent, all with restricted indications. One cytotoxic agent (pemetrexed) with the same restriction as docetaxel in addition to a limitation to nonsquamous non-small cell histology but with a possibility to be used in as single-agent therapy for maintenance. Two tyrosine kinase inhibitors: erlotinib for second-line treatment or for maintenance in case of stable disease after first-line platinum-based chemotherapy, and gefitinib in the presence of an activating mutation on the EGFr gene whatever the line of treatment. One antiangiogenic agent (bevacizumab) for nonsquamous non-small cell tumours in combination with chemotherapy. Those drugs, which are extremely expensive, are not automatically reimbursed by the national social security system, limiting their availability in the routine management of NSCLC.

\section{Which regimen as first-line treatment?}

The recent recommendations of various scientific and academic associations are summarised in table 3. In the fit patient, European guidelines recommend cisplatin-based chemotherapy while American ones propose platinum-based doublets. The cytotoxic drug associated with the platinum derivative should be one of the active compounds listed in table 2, such as gemcitabine, docetaxel, paclitaxel, vinorelbine or pemetrexed. That choice is based on two types of meta-analyses [3]. In the first, the trials are compared according to the number of drugs in the regimen: two-drug regimens are superior to one-drug regimens, both in terms of response and survival; three-drug combinations are better to two drugs only in terms of response but with higher toxicity [4]. In the second type, the role of specific drugs is analysed. Addition of a drug to a platinum derivative is beneficial in term of survival [5].

Non-platinum-based regimens as first-line treatment should be only used in cases where platinum-based chemotherapy is contra-indicated. Indeed, as reported in a meta-analysis of the published literature [11], when all trials were considered (irrespectively of using old or new drugs), there was a significant advantage both for response rate and 1-year survival in favour of platinum-based treatment. The increase in 1 -year survival was $5 \%$. When the analysis was restricted to combination regimens with newer drugs, there was no significant difference in survival but response rate was significantly improved with platinum-based treatment.

There is no demonstration that high dosages of cisplatin (100-120 $\mathrm{mg} \cdot \mathrm{m}^{-2}$ ) provide 
Table 3 Guidelines for the management of advanced NSCLC as proposed by various academic societies and groups

\section{Society}

Cancer Care Ontario Program, 2005 $\& 2010[6]$

European Lung Cancer Working Party (ELCWP), $2006[7]$

\section{First-line therapy}

Platinum-based doublet chemotherapy

Cisplatin-based chemotherapy with one of the regimens shown to be effective

\section{Second-line therapy}

Docetaxel (option: pemetrexed)

followed by erlotinib

Docetaxel (if not already administered as first-line treatment) given on a 3-weekly schedule at a dosage of $75 \mathrm{mg} \cdot \mathrm{m}^{-2}$

American College of Chest Physicians (AACP), 2007 [8]

Platinum-based chemotherapy with a new Should be offered if good PS single agent (ECOG PS 0 -1) pemetrexed
Docetaxel, erlotinib, gefitinib, or
American Society of Clinical Oncology (ASCO), 2009 [9]

Two-drugs platinum-based combination regimen. Clinicians may consider the addition of bevacizumab (based on exclusion criteria of the registration trial) or cetuximab (in patients with an EGFR positive tumour as measured by immunohistochemistry

German Respiratory Society and the German Cancer Society, 2010 [10]
Cisplatin-based combination chemotherapy

PS: performance status
Docetaxel, pemetrexed (non-

squamous carcinoma) and erlotinib better results than standard lower dosages (50$60 \mathrm{mg} \cdot \mathrm{m}^{-2}$ ) in terms of survival. None of the published randomised trials was indeed able to report a significant advantage in favour of high dosages [1]. In fact, the use of high-dose cisplatin is based on an old observation that responders to cisplatin plus vindesine survived longer when $120 \mathrm{mg} \cdot \mathrm{m}^{-2}$ of cisplatin was administered instead of $60 \mathrm{mg} \cdot \mathrm{m}^{-2}$. This difference was observed in a very small group of patients (35 patients). The ELCWP was unable to replicate the results in a much higher number of patients [12]. In addition, a high dosage of cisplatin has the disadvantage of significantly higher renal, auditory and neurological toxicities [13]. Based on all those considerations, standard dosages, associated with reduced toxicity, can thus be recommended.

Cisplatin should be preferred to carboplatin because of a better effect on survival. In randomised trials, the trend is in favour of cisplatin, both in terms of response and survival. The meta-analyses confirm this impression; the results are statistically significant in favour of cisplatin if the analysis is restricted to the regimens using newer drugs combined with platinum derivatives [14-16].

Usually, 4-6 cycles of induction chemotherapy are administered. The level of evidence supporting that attitude is poor, based on a limited number of randomised trials with methodological problems in most of them. Maintenance by prolongation of the treatment after response achievement appears to significantly improve progression-free survival with a modest effect on overall survival [17]. Usually, it is started in the trials after a limited number of cycles of induction chemotherapy. There is, so far, no reported study comparing maintenance after a short induction treatment to induction alone continued until achievement of best response. Nevertheless, the concept of maintenance with a single agent after four cycles of cisplatin-based chemotherapy has been shown associated with survival advantages in comparison with induction only in studies conducted by the pharmaceutical industry. Two drugs have been registered in that indication: pemetrexed [18] for nonprogressing disease and erlotinib [19] for stable disease. Those commercial data have so far not been validated by academic independent trials.

\section{Which treatment at relapse?}

Second-line therapy is today recommended in all guidelines (table 3). This is based on a 
randomised trial having tested second-line chemotherapy versus supportive care alone in advanced NSCLC [20]. Survival was significantly improved in the arm where treatment was chemotherapy. Implementation studies $[21,22]$ have reported results similar to randomised trials when docetaxel was used in routine application. Weekly administration of docetaxel has not been shown to be better [23] than 3-weekly treatment. A dosage of $75 \mathrm{mg} \cdot \mathrm{m}^{-2}$ appears associated with better tolerance than $100 \mathrm{mg} \cdot \mathrm{m}^{-2}$ [24].

For second-line treatment, other drugs have been compared with docetaxel in large randomised trials. They include pemetrexed, erlotinib, gefitinib, vinflunine and oral topotecan. None appeared superior in term of survival and response rate. Two of those drugs have been specifically registered in the European Union for that indication: erlotinib [25] and, in non-squamous NSCLC, pemetrexed [26]. Some improvement was reported with pemetrexed in term of tolerance.

A systematic review of the literature with meta-analysis [27] found a small but significant survival improvement with second-line chemotherapy compared to supportive care alone. The use of drugs other than docetaxel did not improve survival. The authors concluded that docetaxel administered every 3 weeks remains the gold standard.

In conclusion, second-line chemotherapy should be proposed in patients with good performance status, failing after platinum-based first-line chemotherapy. Evidence is in favour of docetaxel (if not administered in first-line treatment) given on a 3-weekly schedule at a dosage of $75 \mathrm{mg} \cdot \mathrm{m}^{-2}$. It should be noted that in routine management, although not supported by good evidence, third-line treatment with active drugs is often proposed in fit patients.

\section{Are there indications for tailored chemotherapy?}

Customised or tailored chemotherapy is a personalised treatment decision based on an analysis of biomarkers of response and resistance to cytotoxic drugs. Customised or tailored chemotherapy results from a treatment decision based on an analysis of biomarkers of response and resistance to cytotoxic drugs. Evidence in favour of that approach comes from post hoc analysis of adjuvant chemotherapy trials in resected NSCLC and from chemotherapy trials where the enzymes RRM1 (regulatory subunit of the ribonucleotide reductase) and

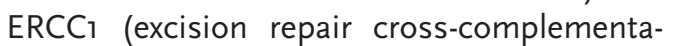
tion group 1) were assessed. If their increased expression was associated with a better prognosis after surgery, a lower expression in the tumour resulted in a better survival for platinum- and gemcitabine-based chemotherapy. The first randomised trial performed on the topic showed improved response rate when treatment was guided by the tumour ERCC1 [28].

Today, there is no validated application of tailored therapy in the routine management of advanced NSCLC, partly because the molecular marker thresholds for selecting treatment have not yet been defined. An indirect approach is to select agents on the basis of the histological subtype. This concept has been used for $>40$ years in the management of lung cancer with the subclassification separating small cell lung cancer from the other subtypes called NSCLC. Recently, there have been newer applications of histology for drug selection with non-squamous NSCLC for pemetrexed and bevacizumab. This has led to a new IASLC/ATS/ERS definition of adenocarcinoma [29]. For bevacizumab, the restriction to non-squamous histology is due to toxicity reason related to haemorrhagic risks in squamous cell carcinoma. For pemetrexed which is a multitarget antifolate, it is based on the fact that the drug is more active on non-squamous tumours which have high activity of enzymes of the folate pathways such as the thymidilate synthase (TS). Those laboratory data could provide an explanation for the findings of the registration trial for NSCLC, which compared cisplatin plus pemetrexed versus cisplatin plus gemcitabine; although no overall difference was found in the trial, survival in the pemetrexed arm improved in non-squamous NSCLC whereas it reduced in squamous cell lung cancer [30]. A retrolective analysis found the same observation in the registered trial performed for second-line therapy. This has led to a restricted indication to non-squamous NSCLC. It should be noted that those survival differences have not been confirmed in a later trial assessing as first-line chemotherapy carboplatin plus pemetrexed versus carboplatin plus gemcitabine [31]. A possible explanation for those discrepancies may be the absence of histology confirmation by an expert panel in all those trials. 


\section{What are the specific indications of targeted therapies?}

Targeted therapies are another way to provide personalised treatment by selecting therapeutic agents according to a target that has been identified on the patient's tumour. There is now a drug marketed in the European Union with that purpose: the tyrosine kinase inhibitor (TKI) gefitinib. Initially developed, as erlotinib, in a patient population unselected for the target, results obtained in control trials were disappointed [32] until the target was identified. The target [33] is located on the receptor of the epithelial growth factor (EGF-R) and is related to activating mutations on exon 19 (deletions) or 21 (L858R point mutation) of the gene. Resistance can be acquired during treatment by secondary EGFR mutation (most commonly, T790M in exon 20) or by the amplification of the MET oncogene.

Some clinical characteristics are associated with the target: adenocarcinoma histology, female sex, nonsmoking and East-Asian ethnicity. It is indeed very frequent in Japan, China and Korea. For that reason, most of the main studies were performed in those countries. In Asiatic non- or ex-smoker patients with adenocarcinoma, gefitinib was shown to be superior to chemotherapy by paclitaxel and carboplatin in term of response rate and progression-free survival [34]. In the subgroup of patients who were positive for EGFR mutation, progression-free survival was significantly longer among those who received gefitinib, while in the subgroup who was negative for the mutation, progression-free survival was significantly longer among those who received carboplatin-paclitaxel. There was no difference in overall survival, probably because there was effective crossover salvage therapy. Those data were confirmed by a trial specifically performed in patients who had a tumour with an activating mutation [35]. The observation that gefitinib is as active in firstline as in salvage treatment has been confirmed in European patients [36].

Based on those data, it is recommended to search for an activating mutation of EGFr, particularly in adenocarcinoma [29] and to treat accordingly the patient by gefitinib, whatever the line of treatment.

\section{How to manage patients in special situations?}

\section{Elderly}

There is no universal definition for elderly without consensual age threshold. Practically, there are three types of situations: the fit patient who can be treated by doublet chemotherapy (although the use of cisplatin has to be very carefully discussed); the fragile patient with significant comorbidities and geriatric syndromes for which anticancer

Table 4 Anticancer treatment in the patient with organ dysfunction and advanced NSCLC

\begin{tabular}{ll} 
Dysfunction & Proposed approach practical approach \\
\hline Renal failure & $\begin{array}{l}\text { Cisplatin and mitomycin are contra-indicated. } \\
\text { Consider carboplatin (prescribed according to renal clearance). }\end{array}$ \\
\hline Liver diseases & Cisplatin-based with drugs nonmetabolised by the liver. \\
\hline Bone marrow & $\begin{array}{l}\text { Chemotherapy with reduced dosages and with active haematological } \\
\text { support. }\end{array}$ \\
\hline Cardiac failure & $\begin{array}{l}\text { Cisplatin, taxans and ifosfamide are contra-indicated. } \\
\text { Consider nonplatinum-based chemotherapy. }\end{array}$ \\
\hline Polyneuropathy & $\begin{array}{l}\text { Cisplatin, vinca-alcaloids and taxans are contra-indicated. } \\
\text { Consider regimens with ifosfamide, gemcitabine, carboplatin or/and } \\
\text { etoposide or TKI. }\end{array}$ \\
\hline Hypoacousia & $\begin{array}{l}\text { Platinum derivatives are contra-indicated. } \\
\text { Consider nonplatinum-based chemotherapy. }\end{array}$
\end{tabular}


treatment is contraindicated; and the patient who has some fragility and is at risk of major deterioration in case of toxic treatment. So far, there are no good tools to separate these three categories and the therapeutic decision is thus mainly based on the clinical judgement.

\section{Poor PS}

Often these patients are mixed in studies with elderly patients, which is a source of confusion. In fact, those patients can be improved if treatment is active, although at the risk of developing significant toxicity [37]. The decision to treat and the type of treatment thus need to be discussed with the patient.

\section{Organ dysfunction}

The main organ dysfunctions that are sources of therapeutic problems are renal failure, hepatic diseases, bone marrow insufficiency, cardiac failure, polyneuropathy and hypoacousia. Here also, there are no good data published [38] and the therapeutic proposal will depend on the cause of the organ dysfunction and of the clinical expertise and judgement of the oncologist. The cause of the organ dysfunction has to be diagnosed, it is important to determine if it is due to the neoplastic disease itself or to another disease. Some practical recommendations are given in table 4 . A good recent review has been published on chemotherapy in case of haemodialysis [39].

\section{Brain metastases}

Brain metastases are not a contra-indication to chemotherapy. In asymptomatic patients, cerebral irradiation can be delayed after cisplatin-based chemotherapy administration [40]. If the extracerebral disease is controlled and the size and the number of brain metastases are limited, stereotaxic radiotherapy should be considered.

\section{Single metastasis}

Local treatment of metastasis by surgery and/or irradiation can be used for symptom control, such as pain, or for avoiding complications, such as bone fractures or respiratory failure due to pleural effusion. In some rare situations, surgical removal or radiosurgery of a single metastasis in the brain [41] or in the adrenals [42] can be curative if the primary tumour has also received curative treatment. 


\section{Educational questions}

1. Which of the following is the best first-line chemotherapy for a fit patient with squamous cell lung cancer and liver metastases?
a. Carboplatin plus gemcitabine
b. Cisplatin plus vinorelbine
c. Cisplatin plus pemetrexed
d. Gefitinib
e. Gemcitabine plus vinorelbine

2. Which treatment would you propose for the above patient in case of moderate renal failure?
a. Carboplatin plus gemcitabine
b. Cisplatin plus vinorelbine
c. Cisplatin plus pemetrexed
d. Gefitinib
e. Gemcitabine plus vinorelbine

3. Among the following drugs, which one is the less active for the treatment of advanced NSCLC?
a. Ifosfamide
b. Epirubicin
c. Docetaxel
d. Vinblastine
e. Gemcitabine

4. A 72-year-old patient with metastatic adenocarcinoma (no mutation for RGFr), ECOG PS 1 and hypoacousia had received first-line chemotherapy with gemcitabine and vinorelbine. He had an objective response but the tumour relapsed 3 months later. Which is the best evidence-based approach?
a. Best supportive care
b. Gefitinib
c. Cisplatin-based chemotherapy
d. Mitomycin plus vindesine
e. Pemetrexed

5. Which one of the following regimens is less adequate for the management of advanced NSCLC as first-line chemotherapy?
a. Cisplatin plus etoposide
b. Cisplatin plus gemcitabine
c. Cisplatin plus mitomycin plus ifosfamide
d. Cisplatin plus vinorelbine
e. Cisplatin plus docetaxel

\section{References}

1. Sculier JP, Moro-Sibilot D. First- and second-line therapy for advanced nonsmall cell lung cancer. Eur Respir J 2009; 33: 915-930.

2. NSCLC Meta-Analyses Collaborative Group. Chemotherapy in addition to supportive care improves survival in advanced non-small-cell lung cancer: a systematic review and meta-analysis of individual patient data from 16 randomized controlled trials. J Clin Oncol 2008; 26: 4617-4625.

3. Meert AP, Sculier JP. What has the meta-analysis contributed to today's standard of care in the treatment of thoracic malignancies? Lung Cancer 2008; 61: 141-151.

4. Delbaldo C, Michiels S, Syz N, et al. Benefits of adding a drug to a single-agent or a 2-agent chemotherapy regimen in advanced non-small-cell lung cancer: a meta-analysis. JAMA 2004; 292: 470-484.

5. Hotta K, Matsuo K, Ueoka H, et al. Addition of platinum compounds to a new agent in patients with advanced non-small-cell lung cancer: a literature based meta-analysis of randomised trials. Ann Oncol 2004; 15: 1782-1789.

6. Goffin J, Lacchetti C, Ellis PM, et al. First-line systemic chemotherapy in the treatment of advanced non-small cell lung cancer: a systematic review. J Thorac Oncol 2010; 5: 260-274.

7. Alexopoulos A, Alard S, Berghmans T, et al. European Lung Cancer Working Party clinical practice guidelines. Non-small cell lung cancer: III. Metastatic disease. Hospital Chronicles 2006; 1: 169-185.

8. Socinski MA, Morris DE, Masters GA, et al. Chemotherapeutic management of stage IV nonsmall cell lung cancer. Chest 2003; 123: 226S-243S.

9. Azzoli CG, Baker S Jr, Temin S, et al. American Society of Clinical Oncology Clinical Practice Guideline update on chemotherapy for stage IV nonsmall-cell lung cancer. J Clin Oncol 2009; 27: $6251-6266$. 
10. Blum T, Schönfeld N, Kollmeier J, et al. Lungenkrebs in Deutschland - zur Versorgungslage der Nation [Lung cancer in Germany: state of the nation]. Pneumologie 2010; 64: 1-12.

11. D'Addario G, Pintilie M, Leighl NB, et al. Platinumbased versus non-platinum-based chemotherapy in advanced non-small-cell lung cancer: a meta-analysis of the published literature. J Clin Oncol 2005; 3: 2926-2936.

12. Klastersky J, Sculier JP, Ravez P, et al. A randomized study comparing a high and a standard dose of cisplatin in combination with etoposide in the treatment of advanced non-small-cell lung carcinoma. J Clin Oncol 1986; 4: 1780-1786.

13. Sculier JP, Klastersky J, Giner V, et al. Phase II randomized trial comparing high-dose cisplatin with moderate- dose cisplatin and carboplatin in patients with advanced non-small-cell lung cancer. European Lung Cancer Working Party. J Clin Oncol 1994; 12: 353-359.

14. Hotta K, Matsuo K, Ueoka H, et al. Meta-analysis of randomized clinical trials comparing Cisplatin to Carboplatin in patients with advanced non-small-cell lung cancer. J Clin Oncol 2004; 22: 3852-3859.

15. Ardizzoni A, Boni L, Tiseo M, et al. Cisplatin- versus carboplatin-based chemotherapy in first-line treatment of advanced non-small-cell lung cancer: an individual patient data meta-analysis. J Nat/ Cancer Inst 2007; 99: 847-857.

16. Jiang J, Liang $X$, Zhou $X$, et al. A meta-analysis of randomized controlled trials comparing carboplatinbased to cisplatin-based chemotherapy in advanced nonsmall cell lung cancer. Lung Cancer 2007; 57: 348-358.

17. Soon YY, Stockler MR, Askie LM, et al. Duration of chemotherapy for advanced non-small-cell lung cancer: a systematic review and meta-analysis of randomized trials. J Clin Oncol 2009; 27: 3277-3283.

18. Ciuleanu T, Brodowicz T, Zielinski C, et al. Maintenance pemetrexed plus best supportive care versus placebo plus best supportive care for nonsmall-cell lung cancer: a randomised, double-blind, phase 3 study. Lancet 2009; 374: 1432-1440.

19. Cappuzzo F, Ciuleanu T, Stelmakh L, et al. Erlotinib as maintenance treatment in advanced non-small-cell lung cancer: a multicentre, randomised, placebo-controlled phase 3 study. Lancet Oncol 2010; 11: 521-529.

20. Shepherd FA, Dancey J, Ramlau R, et al. Prospective randomized trial of docetaxel versus best supportive care in patients with non-small-cell lung cancer previously treated with platinum-based chemotherapy. J Clin Oncol 2000; 18: 2095-2103.

21. Sumpter K, Harper-Wynne C, Yeoh C, et al. Is the second line data on the use of docetaxel in non-small cell lung cancer reproducible ? Lung Cancer 2004; 43: 369-370.

22. Mascaux $\mathrm{C}$, Iannino N, Berghmans $\mathrm{T}$, et al. Le docétaxel comme traitement de rattrapage pour les cancers bronchiques non à petites cellules : étude d'implémentation [Docetaxel as salvage therapy for non-small lung cancer cells: study implementation]. Rev Med Brux 2005; 26: 153-158.

23. Di MM, Perrone F, Chiodini P, et al. Individual patient data meta-analysis of docetaxel administered once every 3 weeks compared with once every week second-line treatment of advanced non-small-cell lung cancer. J Clin Oncol 2007; 25: 1377-1382.

24. Quoix E, Lebeau B, Depierre A, et al. Randomised, multicentre phase II study assessing two doses of docetaxel $\left(75\right.$ or $100 \mathrm{mg} / \mathrm{m}^{2}$ ) as second-line monotherapy for non-small-cell lung cancer. Ann Oncol 2004; 15: 38-44.

25. Shepherd FA, Rodrigues PJ, Ciuleanu T, et al. Erlotinib in previously treated non-small-cell lung cancer. N Engl J Med 2005; 353: 123-132.
26. Hanna N, Shepherd FA, Fossella FV, et al. Randomized phase III trial of pemetrexed versus docetaxel in patients with non-small-cell lung cancer previously treated with chemotherapy. J Clin Oncol 2004; 22: 1589-1597.

27. Tassinari D, Scarpi E, Sartori S, et al. Second-line treatments in non-small cell lung cancer. A systematic review of literature and metaanalysis of randomized clinical trials. Chest 2009; 135: 1596-1609.

28. Cobo M, Isla D, Massuti B, et al. Customizing cisplatin based on quantitative excision repair crosscomplementing 1 mRNA expression: a phase III trial in non-small-cell lung cancer. J Clin Oncol 2007; 25: 2747-2754

29. Travis WD, Brambilla E, Noguchi M, et al. International association for the study of lung cancer/American Thoracic Society/European Respiratory Society international multidisciplinary classification of lung adenocarcinoma. J Thorac Oncol 2011; 6: 244-285.

30. Scagliotti GV, Parikh P, von Pawel J, et al. Phase III study comparing cisplatin plus gemcitabine with cisplatin plus pemetrexed in chemotherapy-naive patients with advanced-stage non-small-cell lung cancer. J Clin Oncol 2008; 26: 3543-3551.

31. Gronberg BH, Bremnes RM, Flotten O, et al. Phase III study by the Norwegian lung cancer study group: pemetrexed plus carboplatin compared with gemcitabine plus carboplatin as first-line chemotherapy in advanced non-small-cell lung cancer. J Clin Oncol 2009; 27: 3217-3224.

32. Thatcher N, Chang A, Parikh P, et al. Gefitinib plus best supportive care in previously treated patients with refractory advanced non-small-cell lung cancer: results from a randomised, placebo-controlled, multicentre study (Iressa Survival Evaluation in Lung Cancer). Lancet 2005; 366: 1527-1537.

33. Cadranel J, Zalcman G, Sequist L. Genetic profiling and epidermal growth factor receptor-directed therapy in nonsmall cell lung cancer. Eur RespirJ 2011; 37: 183-193.

34. Mok TS, Wu YL, Thongprasert S, et al. Gefitinib or carboplatin-paclitaxel in pulmonary adenocarcinoma. N Engl J Med 2009; 361: 947-957.

35. Maemondo M, Inoue A, Kobayashi K, et al. Gefitinib or chemotherapy for non-small-cell lung cancer with mutated EGFR. N Engl J Med 2010; 362: 2380-2388.

36. Rosell R, Moran T, Queralt C, et al. Screening for epidermal growth factor receptor mutations in lung cancer. N Engl J Med 2009; 361: 958-967.

37. Sculier JP, Lafitte JJ, Paesmans M, et al. Chemotherapy improves low performance status lung cancer patients. Eur Respir J 2007; 30: 1186-1192.

38. Lee L, Cheung WY, Atkinson E, et al. Impact of comorbidity on chemotherapy use and outcomes in solid tumors: a systematic review. J Clin Oncol 2011; 29: 106-117.

39. Janus N, Thariat J, Boulanger $\mathrm{H}$, et al. Proposal for dosage adjustment and timing of chemotherapy in hemodialyzed patients. Ann Oncol 2010; 21: 1395-1403.

40. Robinet G, Thomas P, Breton JL, et al. Results of a phase III study of early versus delayed whole brain radiotherapy with concurrent cisplatin and vinorelbine combination in inoperable brain metastasis of non-small-cell lung cancer: Groupe Francais de Pneumo-Cancerologie (GFPC) Protocol 95-1. Ann Oncol 2001; 12: 59-67.

41. Shen KR, Meyers BF, Larner JM, et al. Special treatment issues in lung cancer: ACCP evidencebased clinical practice guidelines (2nd edition). Chest 2007; 132: 290S-305S.

42. Tanvetyanon T, Robinson LA, Schell MJ, et al. Outcomes of adrenalectomy for isolated synchronous versus metachronous adrenal metastases in nonsmall-cell lung cancer: a systematic review and pooled analysis. J Clin Oncol 2008; 26: 1142-1147. 